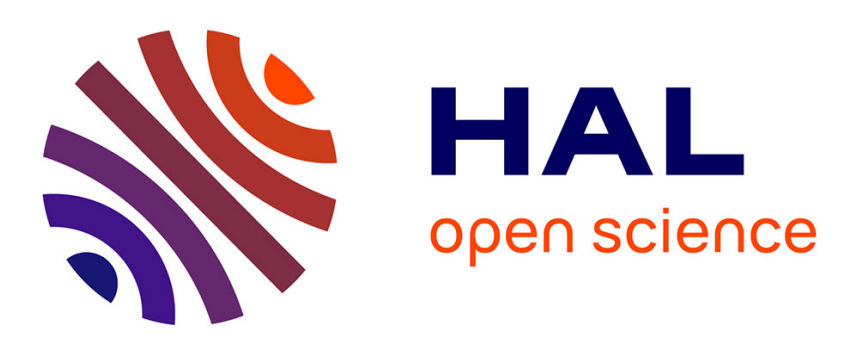

\title{
Improving LoRa Network Capacity Using Multiple Spreading Factor Configurations
}

Dimitrios Zorbas, Georgios Papadopoulos, Patrick Maillé, Nicolas Montavont, Christos Douligeris

\section{- To cite this version:}

Dimitrios Zorbas, Georgios Papadopoulos, Patrick Maillé, Nicolas Montavont, Christos Douligeris. Improving LoRa Network Capacity Using Multiple Spreading Factor Configurations. ICT 2018 - 25th International Conference on Telecommunication, Jun 2018, Saint-Malo, France. pp.1-5, 10.1109/ICT.2018.8464901 . hal-01986487

\section{HAL Id: hal-01986487 https://imt-atlantique.hal.science/hal-01986487}

Submitted on 18 Jan 2019

HAL is a multi-disciplinary open access archive for the deposit and dissemination of scientific research documents, whether they are published or not. The documents may come from teaching and research institutions in France or abroad, or from public or private research centers.
L'archive ouverte pluridisciplinaire HAL, est destinée au dépôt et à la diffusion de documents scientifiques de niveau recherche, publiés ou non, émanant des établissements d'enseignement et de recherche français ou étrangers, des laboratoires publics ou privés. 


\title{
Improving LoRa Network Capacity Using Multiple Spreading Factor Configurations
}

\author{
Dimitrios Zorbas*, Georgios Z. Papadopoulos ${ }^{\dagger}$, Patrick Maille ${ }^{\dagger}$, Nicolas Montavont ${ }^{\dagger}$ and Christos Douligeris* $^{*}$ \\ *NetLab, Department of Informatics, University of Piraeus, Greece, \{dzorbas, cdoulig\}@ unipi.gr \\ ${ }^{\dagger}$ IMT Atlantique, IRISA, UBL, France, \{name.lastname\}@imt-atlantique.fr
}

\begin{abstract}
LoRa networks enable long range communications for Internet of Things (IoT) applications. The current LoRa technology provides a wide range of communication settings whereas many combination settings are orthogonal and, thus, they can be successfully decoded at the gateway when the signals are transmitted simultaneously. Previous simulation results showed that the LoRa network capacity can be improved when multiple communication parameters are applied. In this paper, we model a LoRa network consisting of nodes with different communication settings in terms of bandwidth and spreading factor. We compute the average success probability per configuration as a function of density taking into account both intra and inter-spreading factor collisions. We, also, formulate and solve an optimization problem to maximize the node capacity for a given deployment area and frequency by optimizing the number of nodes having different spreading factor configurations. We present numerical results and we show that solutions close to the optimal can increase the maximum number of nodes by more than $700 \%$ compared to case where equal number of users per spreading factor are considered.
\end{abstract}

\section{INTRODUCTION}

For more than a decade there has been essential interest in designing and deploying IoT applications, with business sectors ranging from smart building and city to smart metering and grid, where low cost and easily deployed IoT devices can provide significant benefits. The IoT technologies are viewed as a cheap and efficient mean for monitoring applications, such as infrastructure monitoring, air quality monitoring or parking in dense urban areas. A shared access network may be the key for new service deployment, where a common infrastructure may be used by different service providers to create new IoT services.

Low-Power Wide-Area Networks (LPWAN) are currently investigated worldwide to allow for low-power operation across a large geographic area. As a result, the interest of the industry, such as Long Range (LoRa) [1], Sigfox [2], RPMA [3] and Weightless [4], towards the LPWAN is gradually increasing. For instance, Sigfox, that operates both as LPWAN technology and as service provider, today covers fourteen countries of Europe ${ }^{1}$. A typical application is the collection of meter readings in a certain geographical area (e.g., city). LPWANs are employed to setup a start network where leaf devices transmit their measurements in 1-hop to a more powerful receiver (i.e., sink or gateway) which then relay the data over an a priori deployed infrastructure to a data collection center.

\footnotetext{
${ }^{1}$ http://www.sigfox.com/en/coverage
}

LoRa is a proprietary spread spectrum modulation technique made by Semtech [1] and is one of the most promising technologies of LPWAN. LoRa is a Layer 1 Network Protocol usually operating on sub-1 $\mathrm{GHz}$ spectrum, in $433 \mathrm{MHz}$, $866 \mathrm{MHz}, 915 \mathrm{MHz}$ frequency bands, while it is agnostic to the used frequency. These frequency bands are unlicensed, which makes the LoRa adoption very straightforward for cities or IoT operators. However, LPWAN's based on LoRa technology still need to be better understood. Previous studies focused on understanding the capacity limits of LoRa networks [5], [6], [7]. Simulation results showed that the LoRa network capacity can be improved when dynamic communication parameters, in terms of bandwidth and spreading factors, are applied. Even if dynamic communication parameters require a centralized entity or a protocol to determine and maintain the nodes settings, a study of such LoRa networks can give some insights on the maximum capabilities of LoRa technology.

In this paper, we model LoRa networks consisting of nodes operating with different combination settings in terms of frequency and spreading factor. For each frequency and for a given deployment distance we are looking for the largest possible number of nodes we could serve with sufficient average success probability. To achieve this, we optimize the portion of nodes of each spreading factor so that the total number of nodes is maximized.

The remainder of this paper is organized as follows. Section II reviews the most pertinent related works from the literature. Section III provides some necessary background for LoRa technology. Section IV presents our model for networks with multiple configurations and the optimization model. In Section V we present numerical results obtained by solving the optimization model. Finally, Section VI concludes the paper and lists future perspectives.

\section{RELATED WORK}

Raza et al. [8] present the five key challenges for LPWAN and compare proprietary and standard technologies, including LoRa, Sigfox, 802.15.4g or Dash7 to cite a few. These challenges are ultra low-power operation and long range communication, since it is expected to cover wide areas for several years. To this aim, 1-hop networks and duty cycling are employed. Low cost is also another challenge for LPWAN. Finally, scalability and quality of service are also challenges given the expected number of connected objects, and the variety of expected services. 
Bor et al. [5] present LoRaSim, a simulator to evaluate the scalability of LoRa networks. The authors detail range of communication options (carrier frequency, spreading factor, bandwidth and coding rate) for a transmitter. Moreover, they study the collision avoidance scenarios as well as the maximum number of transmitters in a LoRa network. Their evaluation results show that to keep the Data Extraction Rate above 0.9, only 120 users are supported per antenna using standard LoRa settings.

Bor et al. [6] also propose LoRaBlink, a MAC and routing cross-layer scheme, above the LoRa physical layer to extend the radio coverage of the gateway. LoRaBlink is self-organized network based on beacons (that contains distance in hops from the sink) and time-slotted channel access method. Their performance evaluation show that LoRaBlink may cover a network of $1.5 \mathrm{ha}$ in a built up environment, achieve $80 \%$ of reliability while having a potential lifetime of 2 years.

Voigt et al. [9] investigate the use of directional antennae and the use of multiple base stations as methods of dealing with internetwork interference. Simulation results show that the use of multiple base stations outperforms the use of directional antennae.

Georgiou and Raza [7] provide a stochastic geometry framework for modeling the performance of a single gateway LoRa network. They model the co-spreading factor interference assuming a single bandwidth frequency for all the nodes and they measure the outage and coverage probabilities based on the signal to noise ratio and the co-spreading sequence interference. Their analysis shows that the coverage probability drops exponentially as the number of end-devices grows due to interfering signals using the same spreading sequence.

Abeele et al. [10] using a LoRa ns-3 module, perform a scalability analysis of LoRaWAN. Their work shows detrimental impact of the downstream traffic on the delivery ratio of the upstream traffic. They, also, show that increasing gateway density can ameliorate but not eliminate this effect, as stringent duty cycle requirements for gateways continue to limit downstream opportunities. The same authors show through simulations that LoRaWAN can send six times more traffic compared to pure Aloha in a single-cell LoRaWAN network for the same number of end devices per gateway when the $125-\mathrm{kHz}$ channel bandwidth is used [11].

In [12], Bankov et al. consider LoRaWAN networks with class A devices operating in acknowledged mode. They detail the data transmission process, considering the difference between power of the signal from different devices and the capture effect. Indeed, they developed a generic mathematical model which can be employed to evaluate network capacity and transmission reliability of LoRaWAN networks when considering Okumura-Hata model for propagation losses.

In this paper, unlike other works, we thoroughly study LoRa performance by presenting both packet success probability as well as the average success probability per frequency and spreading factor configuration as a function of distance and density. We solve an optimization problem for each frequency to find the optimal mix of nodes with different configurations that maximize the density and do not violate a minimum average success probability.

\section{LORA PRELIMINARIES}

\section{A. LoRa Technology}

LoRa is a long-range wireless communication technology, promoted by the LoRa Alliance. At its core, LoRa typically refers to the $i$ ) physical layer: using the Chirp Spread Spectrum (CSS) with integrated Forward Error Correction (FEC); and the ii) MAC layer: the LoRaWAN protocol which operates in a simple star topology and is currently the only available LoRa MAC protocol. A LoRa gateway (sink), such that of Semtech $\mathrm{SX} 1301^{2}$, can receive 8 to 10 concurrent signals with the same carrier frequency as long as these signals are orthogonal (i.e. as they are using different spreading factor settings).

\section{B. Configurable Transmission Parameters}

A LoRa radio comes with five different configuration parameters: i) Transmission Power (TP): the TP on LoRa can be configured from $-4 \mathrm{dBm}$ to $20 \mathrm{dBm}$. Note that European regulations limit the TP to $14 \mathrm{dBm}$ (except of the 869.4-869.65 band which has a $500 \mathrm{~mW}$ limit). ii) Carrier Frequency (CF): typically, LoRa may operate on sub- $1 G H z$ and, thus, can be set between $137 \mathrm{MHz}$ and $1020 \mathrm{MHz}$, note that $\mathrm{CF}$ is the center frequency with steps of $61 \mathrm{~Hz}$. iii) Bandwidth $(\boldsymbol{B W})$ : in LoRa the frequencies width can be configured between $7.8 \mathrm{kHz}$ to $500 \mathrm{kHz}$, however, the most popular options are $500 \mathrm{kHz}, 250 \mathrm{kHz}$ and $125 \mathrm{kHz}$. Note that higher $\mathrm{BW}$ values allow higher data rates and, thus, shorter airtime. On the other hand, lower BW values allow higher sensitivity, but with lower data rate. Very low BWs (i.e., below $62.5 \mathrm{kHz}$ ) require a temperature compensated crystal oscillator. $i v$ ) Coding Rate (CR): LoRa employs Forward Error Correction (FEC) to overcome the potential interferences, thus, the CR is the FEC rate (i.e., 4/5, 4/6, 4/7 and 4/8). The higher the $C R$, the more robust the transmission, at the cost of the increased air time. v) Spreading Factor $(\boldsymbol{S F})$ : SF defines the number of chirps per symbol. SF can be configured from 6 to 12. The higher the SF, the higher the receiver sensitivity which allows longer communication ranges but, also, higher packet airtime. The radio communications under different SF are orthogonal to each other and, thus, the network separation by employing different $\mathrm{SF}$ is possible [7], [5].

\section{Conditions for obtaining a Collision}

In LoRa networks, when two or more concurrent transmissions arrive at the receiver, we may observe a collision under certain conditions [5]. More specifically, the two transmissions: $i)$ should overlap in arrival time, ii) should overlap in Carrier Frequency, otherwise the gateway may decode both transmissions (i.e., if it is listening at both carrier frequencies), iii) should overlap in Spreading Factors, since the SF are orthogonal in LoRa, the receiver may decode if it receives at

\footnotetext{
${ }^{2}$ http://www.semtech.com/apps/filedown/down.php?file=sx1301.pdf
} 
different SFs, $\boldsymbol{i} v)$ if the difference in received signal strength is negligible, then the receiver is not able to decode efficiently either transmission. $v$ ) Finally, in LoRa, we may observe the capture effect, when two transmissions arrive at the gateway and the weaker signal is suppressed by the stronger.

\section{LORA SCALABILITY MODEL}

\section{A. Preliminaries}

Let us assume that $k$ packets are sent in a period of time $\tau$ and $T_{p k t}$ is the transmission time given by the following equation [13]:

$$
\begin{aligned}
& T_{p k t}=\left(n_{p}+4.25\right) \frac{2^{S F}}{B W}+ \\
& \left(8+\max \left(\operatorname{ceil}\left(\frac{8 P L-4 S F+28+16-20 H}{4(S F-2 D E)}\right)(C R+4), 0\right)\right) \frac{2^{S F}}{B W},
\end{aligned}
$$

where $n_{p}$ is the number of programmed preamble symbols, $P L$ is the packet payload, $H=0$ when the header is enabled and $H=1$ when no header is present. $D E=1$ when the low data rate optimization is enabled and $D E=0$ for disabled.

Moreover, a signal can be decoded at the gateway only when its power $\left(P_{r x}\right)$ is higher than a transceiver sensitivity $\left(S^{S F, B W}\right)$ as it is defined by the corresponding combination of spreading factor - bandwidth:

$$
P_{r x}>S^{S F, B W},
$$

where $P_{r x}$ is given by the following formula:

$$
P_{r x}=P_{t x}+G-L-L_{p l} .
$$

$P_{t x}$ is the transmission power, $G$ is the antenna power gains and $L$ is the power losses at the transmitter. $L_{p l}$ describes the attenuation of the signal in relation with the distance $d$ :

$$
L_{p l}=L_{p l}^{d_{0}}+10 \gamma \ln \frac{d}{d_{0}}-X_{\sigma},
$$

$L_{p l}^{d_{0}}$ is the received power at reference distance $d_{0}, \gamma$ is the path-loss factor, and $X_{\sigma}$ is the variance.

\section{B. Success probability}

Our goal in this section is to express the maximum user density a gateway can cover with a given frequency, assuming that users are distributed uniformly over the disk of radius $d$ centered at the gateway. We consider that nodes with multiple SF (operating at the same frequency) may be placed in this disk. We take into account both intra and inter-SF eventual collisions caused by transmissions using the same SF or frequency. We model intra and inter-SF collisions using the following rules:

1) Intra-SF collisions: a collision occurs between two LoRa frames with the same SF and frequency. In this case, only the LoRa frame with the highest power can be decoded and its power at the gateway exceeds the other one minus $6 \mathrm{~dB}$.

2) Inter-SF collisions: a collision occurs between two LoRa frames with the same frequency and different SF. In this case, the first frame is demodulated if the received power of the first minus the received power of the second is
TABLE I

MINIMUM SINR REQUIRED TO DEMODULATE PER SPREADING FACTOR (SF) [14]

\begin{tabular}{|c|c|}
\hline SF & Min SINR (dB) \\
\hline 7 & -7 \\
8 & -9 \\
9 & -11.5 \\
10 & -14 \\
11 & -16.5 \\
12 & -19 \\
\hline
\end{tabular}

higher than the Signal-to-Interference-plus-Noise Ratio (SINR) of the first one (see Table I).

We treat the LoRa layer as a protocol without retransmissions and we assume that the nodes emit packets independently of each other and of their location with an individual intensity $\theta=k / \tau$.

We denote by $D$ the density of nodes in the disk, so that, assuming $D$ large enough:

- the total number of nodes $N$ for the given frequency is $D \pi d^{2}$

- the transmissions from nodes within a distance $\leq d$ from the gateway follow a Poisson distribution with rate $\theta D \pi d^{2}$.

In the rest of this section, we only consider a single SF/BW configuration. Now, let us consider a node at distance $x$ from the gateway, emitting a message. The emission is successful if no other node's emission overlaps with the current emission and no intra or inter-SF collisions happen. Considering fixed environmental conditions and all nodes have the same transmission parameters, only the distance counts, so using Eq. $(3,4)$ the potential intra-SF interferers are those whose distance from the gateway is below $x R$ with $R:=e^{\frac{6}{10 \gamma}}>1$. The number of potential interferers depends on the number of nodes with the same SF and BW. We denote with $\alpha_{i}$ the percentage of nodes in $N$ having $\mathrm{SF}$ equal to $\mathrm{SF}_{i}, i \in[7,12]$. It holds that $\sum_{i=7}^{12} \alpha_{i}=1$. We, also, denote with $D_{i}$ the density of the nodes having $\mathrm{SF}$ equal to $\mathrm{SF}_{i}$, with $D_{i}=\frac{\alpha_{i} N}{\pi d^{2}}$.

On the other hand, the amount of nodes with the same $\mathrm{SF}_{i}$ may interfere with nodes having different SF but transmitting in the same frequency. Following the second collision rule and using Eq. $(3,4)$ the potential inter-SF interferers are those whose distance from the gateway is below $x Q$ with $Q:=e^{\frac{S I N R_{i}}{10 \gamma}}$, where $S I N R_{i}$ is the corresponding SINR of transmissions conducted using $\mathrm{SF}_{i}$.

Summarizing, the number of potential interferers is $D_{i} \pi(R x)^{2}+D \pi(Q x)^{2}$, and the probability of successful transmission $P_{\text {success }}(x)$ is therefore the probability that within a vulnerability period of duration $2 T_{p k t}$, none of those potential interfering nodes starts a transmission. We, hence, have

$$
P_{\text {success }}(x):=e^{-2 T_{p k t} \theta\left(D_{i} \pi(R x)^{2}+D \pi(Q x)^{2}\right)}
$$

It is easy to observe that when $x \rightarrow 0, P_{\text {success }} \rightarrow 1$ (best case scenario), while the worst case scenario appears when $x \rightarrow d$.

Finally, we compute the average success probability among nodes. Note that some nodes will have a success probability 
below the average, but we expect that because of a potential node mobility, that success probability will evolve over time so that the individual time-average equals the average among nodes. With a uniform distribution of nodes over the disk with density $D$, the infinitesimal number of users having a spreading factor $i$ within distance $[x, x+\mathrm{d} x]$ from the gateway is $2 x D_{i} \pi \mathrm{d} x$, so that the average success probability among all nodes in the disk is given by the following equation:

$$
\begin{aligned}
P_{\mathrm{avg}}^{i} & :=\frac{1}{D_{i} \pi d^{2}} \int_{x=0}^{d} 2 x D_{i} \pi P_{\text {success }}(x) \mathrm{d} x \\
& :=\frac{e^{-2 T_{p k t} \theta \pi d^{2}\left(D_{i} R^{2}+D Q^{2}\right)}-1}{-2 T_{p k t} \theta \pi d^{2}\left(D_{i} R^{2}+D Q^{2}\right)}
\end{aligned}
$$

Given $\alpha_{i}$, finding the maximum density $D$ such that $P_{\text {avg }}^{i}$ is above a threshold looks intractable analytically, but can easily be done numerically.

\section{Optimal capacity settings}

In this subsection we formulate a capacity maximization problem for a given frequency (i.e., BW), deployment distance $d$, and packet rate (i.e., $\theta$ ) as a function of a minimum achievable average success probability $P_{\min }$. The problem is transformed to a problem of finding the optimal percentage of nodes for each SF (i.e., $\alpha_{i}$ ) such that the density of the nodes having the same frequency is maximized.

$$
\max N
$$

s.t.

$$
\begin{gathered}
\frac{e^{-2 T_{p k t} \theta \pi d^{2}\left(D_{i} R^{2}+D Q^{2}\right)}-1}{-2 T_{p k t} \theta \pi d^{2}\left(D_{i} R^{2}+D Q^{2}\right)} \geq P_{\text {min }}, \forall i \in[7,12], \\
T_{p k t} \text { by Eq. }(1), \\
R=e^{\frac{6}{10 \gamma}}, \\
Q=e^{\frac{S I N R_{i}}{10 \gamma}}, \forall i \in[7,12], \\
D_{i}=\frac{\alpha_{i} N}{\pi d^{2}} \\
D=\frac{N}{\pi d^{2}}, \\
\alpha_{i} \in[0, \chi, 2 \chi, \ldots, 1], \forall i \in[7,12] \\
0 \leq \chi \leq 1, \chi \in \mathcal{R}, \\
\sum_{i=7}^{12} \alpha_{i}=1 .
\end{gathered}
$$

Note that the optimal solution can be approximated by considering discrete values for all $\alpha_{i}$ 's. The smaller the step $\chi$ between two concurrent values, the better the approximation.

\section{Performance Evaluation}

\section{A. LoRa and network configuration}

To evaluate the proposed model we used LoRa and network parameters as they appear in Table II [5]. We assume that all the nodes are placed within the given distance limits and are positioned around a single gateway. The evaluation was conducted using a set of Python scripts that implement Eq. (5) and (6). We also solve the optimization problem of (7) using exhaustive search and considering $\chi=0.01$ for all $\alpha$ values. A minimum success probability of 0.9 and a maximum deployment distance
TABLE II

LORA AND NETWORK PARAMETERS

\begin{tabular}{|ll|}
\hline Parameter & Value \\
\hline Coding Rate $(\mathrm{CR})$ & $4 / 5$ \\
Bandwidth (BW) & $\{125,250,500\} k H z$ \\
Spreading Factor (SF) & $7-12$ \\
Sensitivity per SF and BW & same as Table 1 of [5] \\
Carrier Frequency (CF) & $868 \mathrm{MHz}$ \\
Payload (PL) & 20 bytes \\
Low data rate optimization $(\mathrm{DE})$ & 1 for SF $=\{11,12\}$ \\
& and BW=125, 0 otherwise \\
Header $(\mathrm{H})$ & 0 \\
Preamble symbols $\left(n_{p}\right)$ & 8 \\
Transmission power $\left(P_{t x}\right)$ & $14 \mathrm{dBm}$ \\
Gains minus losses $(G-L)$ & 0 \\
Path loss exponent $(\gamma)$ & 4 \\
Reference distance $\left(d_{0}\right)$ & $40 \mathrm{~m}$ \\
Max. distance to gateway $(d)$ & $100 \mathrm{~m}$ \\
Power at reference distance $\left(L_{p l}^{d_{0}}\right)$ & $127.41 \mathrm{dBm}$ \\
Variance $\left(X_{\sigma}\right)$ & $N(0,1)$ \\
Packet interval $\tau$ & $200-1000$ secs \\
Packet rate $\theta$ & 1 packet per $\tau(k=1)$ \\
\hline
\end{tabular}

of $100 \mathrm{~m}$ were also considered. This distance was chosen so that all the nodes regardless their SF settings will be able to reach the gateway.

\section{B. Numerical results}

Table III presents the best $\alpha$ values for different bandwidth frequencies and packet rates. The results show that for all the cases the capacity is maximized when the following combination of SF happens: $\alpha_{7}=0.77, \alpha_{8}=0.23$, and $\alpha_{9}=\alpha_{10}=$ $\alpha_{11}=\alpha_{12}=0$. This means that only a mix of nodes having $\mathrm{SF}=7$ and $\mathrm{SF}=8$ provides the best result.

Figures 1, 2 and 3 illustrate the maximum number of users per BW and packet rate. An equal number of $\alpha$ was used in the first figure (thus equal numbers of users per SF), while in the second figure the algorithm includes only nodes with $\mathrm{SF}=7$. Finally, the values of Table III were used in the second figure. The results show that considering close to optimal settings the potential number of users can be increased by up to $705 \%$ and up to $16 \%$ for the cases of Figure 1 and 2 respectively. Indeed, on one hand, by increasing the number of users having higher SFs, the time on air (i.e., $T_{p k t}$ ) increases significantly and, thus, there is a higher probability of intra and inter-SF collisions. On the other hand, the number of intra-SF collisions may be also high if a high number of nodes with the same SF is deployed.

\section{CONCLUSION \& FUTURE WORK}

In this paper we modeled a LoRa network comprised from nodes with variable spreading factor and frequency combinations. Considering both intra and inter-SF collisions, we modeled the packet generation process of each combination as a Poisson process and we computed the average packet success probability. We, also, formulated a capacity optimization problem for a given circular deployment area and a minimum allowed probability of successful packet delivery. We solved this problem with a good approximation taking into account different populations and maximum packet rated for each of the 
TABLE III

BEST $\alpha$ VALUES TO MAXIMIZE CAPACITY

\begin{tabular}{|cc|cccccc|}
\hline$\theta(p k t / s e c)$ & $\mathrm{BW}$ & $\alpha_{7}$ & $\alpha_{8}$ & $\alpha_{9}$ & $\alpha_{10}$ & $\alpha_{11}$ & $\alpha_{12}$ \\
\hline $1 / 200$ & 125 & 0.77 & 0.23 & 0 & 0 & 0 & 0 \\
$1 / 200$ & 250 & 0.77 & 0.23 & 0 & 0 & 0 & 0 \\
$1 / 200$ & 500 & 0.77 & 0.23 & 0 & 0 & 0 & 0 \\
\hline $1 / 300$ & 125 & 0.77 & 0.23 & 0 & 0 & 0 & 0 \\
$1 / 300$ & 250 & 0.77 & 0.23 & 0 & 0 & 0 & 0 \\
$1 / 300$ & 500 & 0.77 & 0.23 & 0 & 0 & 0 & 0 \\
\hline $1 / 400$ & 125 & 0.77 & 0.23 & 0 & 0 & 0 & 0 \\
$1 / 400$ & 250 & 0.77 & 0.23 & 0 & 0 & 0 & 0 \\
$1 / 400$ & 500 & 0.77 & 0.23 & 0 & 0 & 0 & 0 \\
\hline $1 / 500$ & 125 & 0.77 & 0.23 & 0 & 0 & 0 & 0 \\
$1 / 500$ & 250 & 0.77 & 0.23 & 0 & 0 & 0 & 0 \\
$1 / 500$ & 500 & 0.77 & 0.23 & 0 & 0 & 0 & 0 \\
\hline $1 / 600$ & 125 & 0.77 & 0.23 & 0 & 0 & 0 & 0 \\
$1 / 600$ & 250 & 0.77 & 0.23 & 0 & 0 & 0 & 0 \\
$1 / 600$ & 500 & 0.77 & 0.23 & 0 & 0 & 0 & 0 \\
\hline $1 / 700$ & 125 & 0.77 & 0.23 & 0 & 0 & 0 & 0 \\
$1 / 700$ & 250 & 0.77 & 0.23 & 0 & 0 & 0 & 0 \\
$1 / 700$ & 500 & 0.77 & 0.23 & 0 & 0 & 0 & 0 \\
\hline $1 / 800$ & 125 & 0.77 & 0.23 & 0 & 0 & 0 & 0 \\
$1 / 800$ & 250 & 0.77 & 0.23 & 0 & 0 & 0 & 0 \\
$1 / 800$ & 500 & 0.77 & 0.23 & 0 & 0 & 0 & 0 \\
\hline $1 / 900$ & 125 & 0.77 & 0.23 & 0 & 0 & 0 & 0 \\
$1 / 900$ & 250 & 0.77 & 0.23 & 0 & 0 & 0 & 0 \\
$1 / 900$ & 500 & 0.77 & 0.23 & 0 & 0 & 0 & 0 \\
\hline $1 / 1000$ & 125 & 0.77 & 0.23 & 0 & 0 & 0 & 0 \\
$1 / 1000$ & 250 & 0.77 & 0.23 & 0 & 0 & 0 & 0 \\
$1 / 1000$ & 500 & 0.77 & 0.23 & 0 & 0 & 0 & 0 \\
\hline & & & & & & & \\
\hline
\end{tabular}

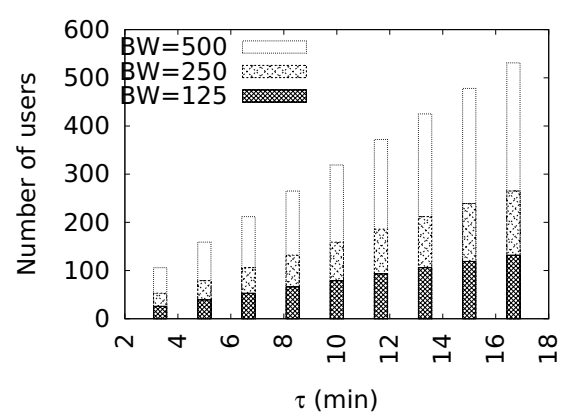

Fig. 1. Maximum number of users per frequency with $\alpha_{7}=\alpha_{8}=\alpha_{9}=$ $\alpha_{10}=\alpha_{11}=\alpha_{12}=1 / 6$.

available SF/BW configurations. Our numerical results showed that the number of LoRa users using close to optimal settings can be increased by more than $700 \%$ compared to the case where equal node populations per SF are considered or or up to $16 \%$ compared to the case where only nodes with the minimum possible packet air time are deployed.

Part of our future work is to find optimal node distributions within each SF/BW configuration, discarding the current uniform node placement assumption. Optimizing the nodes' density, their number in the system can be further maximized.

\section{ACKNOWLEDGMENTS}

This work was carried out within the action "Strengthening Post Doctoral Research" of the "Human Resources Development Program, Education and Lifelong Learning”, 2014-2020, which is being implemented from IKY and is co-financed by the European Social Fund - ESF and the Greek government.

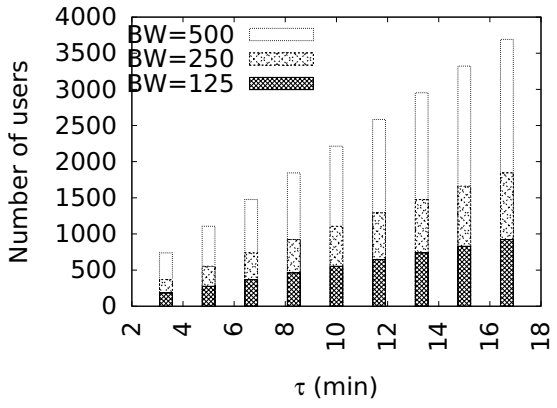

Fig. 2. Maximum number of users per frequency with $\alpha_{7}=1$ and $\alpha_{8}=$ $\alpha_{9}=\alpha_{10}=\alpha_{11}=\alpha_{12}=0$.

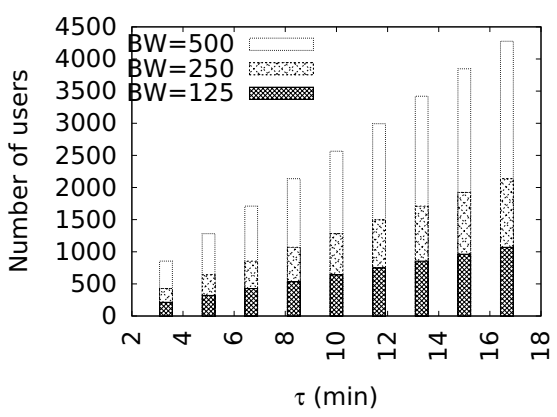

Fig. 3. Maximum number of users per frequency with the best $\alpha$ values found solving the optimization problem.

\section{REFERENCES}

[1] "LoRa Alliance web page," 2016, https://www.lora-alliance.org/.

[2] "Sigfox - the global communications service provider for the internet of things (iot)," https://www.sigfox.com/fr, (Accessed on 01/27/2017).

[3] "Rpma technology - ingenu," http://www.ingenu.com/technology/rpma/, (Accessed on 01/27/2017).

[4] "Weightless - setting the standard for iot," http://www.weightless.org/, (Accessed on 01/27/2017).

[5] M. C. Bor, U. Roedig, T. Voigt, and J. M. Alonso, "Do lora low-power wide-area networks scale?" in Proceedings of the 19th ACM International Conference on Modeling, Analysis and Simulation of Wireless and Mobile Systems, ser. MSWiM '16. New York, NY, USA: ACM, 2016, pp. 59-67.

[6] M. Bor, J. Vidler, and U. Roedig, "Lora for the internet of things," in Proceedings of the 2016 International Conference on Embedded Wireless Systems and Networks, ser. EWSN '16. USA: Junction Publishing, 2016, pp. 361-366.

[7] O. Georgiou and U. Raza, "Low power wide area network analysis: Can lora scale?" IEEE Wireless Communications Letters, 2017.

[8] U. Raza, P. Kulkarni, and M. Sooriyabandara, "Low power wide area networks: A survey," CoRR, vol. abs/1606.07360, 2016.

[9] T. Voigt, M. Bor, U. Roedig, and J. Alonso, "Mitigating inter-network interference in lora networks," arXiv preprint arXiv:1611.00688, 2016.

[10] F. V. d. Abeele, J. Haxhibeqiri, I. Moerman, and J. Hoebeke, "Scalability analysis of large-scale lorawan networks in ns-3," arXiv preprint arXiv:1705.05899, 2017.

[11] J. Haxhibeqiri, F. Van den Abeele, I. Moerman, and J. Hoebeke, "Lora scalability: A simulation model based on interference measurements," Sensors, vol. 17, no. 6, 2017.

[12] D. Bankov, E. Khorov, and A. Lyakhov, "Mathematical Model of LoRaWAN Channel Access with Capture Effect," in Proceedings of the 28th Annual IEEE International Symposium on Personal, Indoor and Mobile Radio Communications (PIMRC), 2017.

[13] "Semtech. an1200.13 sx1272/3/6/7/8: Lora modem - designer's guide, revision 1," July 2013.

[14] N. Varsier and J. Schwoerer, "Capacity limits of lorawan technology for smart metering applications," in 2017 IEEE International Conference on Communications (ICC), May 2017, pp. 1-6. 